\title{
Risco e ocorrência de quedas em idosos atendidos na Estratégia da Saúde da Família no interior de Mato Grosso
}

\author{
Risk and occurrence of falls in elderly care in Family Health Strategy inside Mato Grosso \\ Riesgo y ocurrencia de caídas en el cuidado de mayores en la Estrategia de Salud Familiar dentro \\ de Mato Grosso
}

Recebido: 02/04/2021 | Revisado: 09/04/2021 | Aceito: 13/04/2021 | Publicado: 23/04/2021

Suelly Lavinia Venceslau de Almeida Moraes
ORCID: https://orcid.org/0000-0001-7490-3374
Universidade do Estado de Mato Grosso, Brasil
E-mail: suellylavinia18@gmail.com
Alexandra de Paula Rothebarth
ORCID: https://orcid.org/0000-0001-9053-3166
Universidade Federal de Mato Grosso, Brasil
E-mail: ale_rothebarth@ hotmail.com
Daniela Luzia Zagoto Agulhó
ORCID: https://orcid.org/0000-0002-3434-9863
Universidade Federal de Mato Grosso, Brasil
E-mail: dani.zagoto@ outlook.com
Karina Nonato Mocheuti
ORCID: https://orcid.org/000-0002-1800-2640
Universidade do estado de Mato Grosso, Brasil
E-mail: enfkarinanonato@gmail.com

\begin{abstract}
Resumo
Os acidentes por quedas constituem um importante agravo à saúde da população idosa, representando um problema de saúde pública. Objetivo: Descrever o perfil da população idosa e identificar a ocorrência de quedas e o risco de cair de idosos da comunidade atendidos por uma equipe da Estratégia da Saúde da Família, num munícipio da região Norte Mato-grossense. Métodos: Pesquisa transversal, realizada em 2019, com 50 idosos acompanhados na atenção básica, que atenderam aos critérios de inclusão. Utilizou-se um questionário sóciodemográfico e o Escore de Risco de Quedas traduzido e validado para o Brasil. Posteriormente, aplicou-se a técnica estatística de análise descritiva, apresentada em tabelas e gráfico comparativos, com frequências absolutas e relativas, e posteriormente foi feita a discussão com base na literatura sobre o assunto pesquisado. Resultados: Os participantes eram idosos jovens, com prevalência do sexo feminino, casados, aposentados e/ou pensionistas, não etilista e tabagistas. A maioria residia com familiares, 26\% tinham de 1 a 4 anos de estudo e $26 \%$ de 9 a 10 anos. $46 \%$ tinham renda mensal de até 1 salário mínimo; 92\% relataram comorbidades; 54\% negaram praticar atividade física. Observou-se que $68 \%$ possuíam alto risco de sofrerem quedas, 74\% referiram ter caído após 60 anos e, $73 \%$ a queda foi decorrente de escorregão/tropeço. A residência foi o principal local de ocorrência de quedas e, 37,8\% dos idosos apresentaram fraturas. Conclusão: A prevalência do risco de quedas é elevada. Faz-se necessário delinear estratégias para prevenção de quedas nos idosos e refletir sobre a necessidade de formação continuada para a equipe da ESF, em especial o enfermeiro.
\end{abstract}

Palavras-chave: Envelhecimento; Acidentes por quedas; Atenção primária à saúde.

\begin{abstract}
Falls accidents are an important health problem for the elderly population, representing a public health problem. Objective: To describe the profile of the elderly population and to identify the occurrence of falls and the risk of falling for elderly people in the community assisted by a Family Health Strategy team, in a municipality in the northern region of Mato Grosso. Methods: Cross-sectional survey, conducted in 2019, with 50 elderly people followed up in primary care, who met the inclusion criteria. A socio-demographic questionnaire and the Falls Risk Score translated and validated for Brazil were used. Subsequently, the statistical technique of descriptive analysis was applied, presented in comparative tables and graphs, with absolute and relative frequencies, and later the discussion was made based on the literature on the researched subject. Results: The participants were young elderly, with a prevalence of females, married, retired and / or pensioners, non-alcoholic and non smokers. Most lived with family members, $26 \%$ had 1 to 4 years of study and $26 \%$ had 9 to 10 years. $46 \%$ had a monthly income of up to 1 minimum wage; $92 \%$ reported comorbidities; $54 \%$ denied practicing physical activity. It was observed that $68 \%$ had a high risk of falling, 74\% reported having fallen after 60 years and, $73 \%$ the fall was due to slipping / stumbling. The residence was the main place of occurrence of falls and, $37.8 \%$ of the elderly had fractures. Conclusion: The prevalence of risk
\end{abstract}


of falls is high. It is necessary to outline strategies to prevent falls in the elderly and reflect on the need for continued training for the FHS team, especially the nurse.

Keywords: Aging; Accidents due to falls; Primary health care.

\section{Resumen}

Los accidentes por caídas son un problema de salud importante para la población anciana, representando un problema de salud pública. Objetivo: Describir el perfil de la población anciana e identificar la ocurrencia de caídas y el riesgo de caídas de personas mayores en la comunidad asistidas por un equipo de Estrategia de Salud de la Familia, en un municipio del Norte de Mato Grosso. Métodos: Encuesta transversal, realizada en 2019, con 50 adultos mayores seguidos en atención primaria, que cumplieron con los criterios de inclusión. Se utilizó un cuestionario sociodemográfico y el Score de Riesgo de Caídas traducido y validado para Brasil. Posteriormente, se aplicó la técnica estadística de análisis descriptivo, presentada en tablas y gráficos comparativos, con frecuencias absolutas y relativas, y posteriormente se realizó la discusión con base en la literatura sobre el tema investigado. Resultados: Los participantes fueron adultos jóvenes, con predominio del sexo femenino, casados, jubilados y / o pensionados, no alcohólicos y fumadores. La mayoría vivía con miembros de la familia, el 26\% tenía de 1 a 4 años de estudio y el $26 \%$ tenía de 9 a 10 años. El 46\% tenía un ingreso mensual de hasta 1 salario mínimo; 92\% informó comorbilidades; El $54 \%$ negó practicar actividad física. Se observó que el 68\% tenía un alto riesgo de caída, el $74 \%$ informó haber caído después de 60 años y el 73\% la caída se debió a resbalones / tropiezos. La residencia fue el principal lugar de ocurrencia de caídas y el 37,8\% de los ancianos presentó fracturas. Conclusión: la prevalencia de riesgo de caídas es alta. Es necesario delinear estrategias para prevenir caídas en el anciano y reflexionar sobre la necesidad de una formación continua del equipo de la ESF, especialmente del enfermero.

Palabras clave: Envejecimiento; Accidentes por caídas; Primeros auxilios.

\section{Introdução}

O aumento da expectativa de vida é uma realidade vivenciada mundialmente. No Brasil o envelhecimento populacional é relativamente recente (século XX). Esse cenário implicou no surgimento de movimentos sociais voltados para a construção de políticas de saúde para a população idosa (Veras; Oliveira, 2018). Conforme dados do Instituto Brasileiro de Geografia e Estatística (IBGE), estima-se que no ano de 2060 a população idosa representará $25,5 \%$, ou seja, um quarto da população brasileira (Brasil, 2018).

O envelhecimento é um processo sequencial e individual, e por isso pode variar de indivíduo para indivíduo, sendo gradativo para alguns e acelerado para outros. Esses efeitos são condicionados por fatores como o estilo de vida, condições socioeconômicas, doenças crônicas, não podendo, ainda, desconsiderar o fator biológico que se caracteriza pelos planos molecular, celular, tecidual e orgânico do indivíduo (Azevedo, Azevedo \& Istoe, 2018).

Mesmo no envelhecimento saudável, pode ser que alguns idosos sofram determinado grau de comprometimento fisiológico. O que pode desencadear o aumento da vulnerabilidade e fragilidade pela diminuição de massa muscular, comprometimento do equilíbrio, alterações de marcha e mobilidade, ampliando a probabilidade de ocorrência de eventos indesejados, como os acidentes por quedas, exigindo novas abordagens dos sistemas de saúde (Chagas; Rocha, 2012). Entende-se por quedas o deslocamento não intencional ao solo ou em nível inferior, não levando em consideração possíveis mudanças de posição intencional para apoiar-se (Organização Mundial da Saúde - OMS, 2010).

Segundo a OMS, de $28 \%$ a $35 \%$ das pessoas com mais de 65 anos de idade sofrem algum episódio de queda a cada ano. A proporção se eleva para valores que oscilam de 32\% a $42 \%$ para os idosos com mais de 70 anos, alcançando $50 \%$ em idosos com 80 anos ou mais (OMS, 2012).

A queda do idoso é um importante indicativo de síndrome geriátrica, enquanto evento multifatorial e heterogêneo, que pode gerar diversas consequências físicas e psicológicas (Ferreira et.al., 2019). Isso implica necessidade de rever a crença, em especial dos idosos, de que as quedas são inerentes ao processo de envelhecimento, para que se possa melhor conhecer, suprimir ou mitigar os fatores de risco (Morsch, Myskiw \& Myskiw, 2016).

A ocorrência das quedas é decorrente da combinação entre fator intrínseco que está diretamente ligado ao próprio indivíduo, o qual, com o avançar da idade, sofre declínio da função motora e cognitiva (Oliveira, Baixinho \& Henrique, 2018); 
e fatores extrínsecos ligados ao meio externo e à estrutura física dos locais nos quais os idosos habitam e realizam suas atividades diárias.

Considerando esses fatores, intrínsecos e extrínsecos, foi criado o Comitê Assessor de Políticas de Prevenção e Promoção dos Cuidados da Osteoporose e de Quedas na População Idosa, colegiado responsável por apoiar as políticas do Ministério da Saúde voltadas para a população idosa, no que diz respeito a osteoporose e ao evento queda, objetivando reduzir a internação e tempo de permanência hospitalar. Na Portaria de constituição desse comitê é expressamente reconhecido o papel fundamental dos profissionais da atenção básica, em especial o enfermeiro, na implantação dessas políticas (Brasil, 2007).

Desta forma, o atendimento ofertado pelo enfermeiro no cuidado a esta população é multifacetado e inclui ações de promoção a saúde, educação em saúde, prevenção, assistência domiciliar, consultas de enfermagem com planejamento da assistência voltadas a singularidade desta população como risco a quedas, dentre outros (Amthauer \& Falk, 2017).

Assim, cabe ao enfermeiro formular e executar as atividades de educação em saúde para sensibilizar os idosos de que o evento da queda é evitável, propondo medidas para identificar, suprimir e/ou mitigar fatores de risco; bem como desenvolver capacitação permanente da equipe que atende esta população (Gasparoto, Falsarella \& Coimbra, 2014).

Dado esse contexto mostra-se relevante identificar o risco de queda a que estão sujeitos os idosos de um município da região Norte Mato-grossense, para que possam ser planejadas ações educacionais e promotoras de saúde, capazes de estimular o autocuidado e, subsidiar o trabalho de enfermeiros e da equipe da Estratégia Saúde da Família (ESF) no que concerne ao desenvolvimento de estratégias que beneficiem essa população. Dessa forma, o objetivo deste estudo foi descrever o perfil da população idosa de um município da região Norte Mato-grossense e identificar a ocorrência de quedas e o risco de cair.

\section{Metodologia}

Pesquisa do tipo transversal, de cunho descritivo e com abordagem quantitativa (Zangirolami-Raimundo, Echeimberg, Leone, 2018). Foi realizada em uma ESF de uma cidade da região Norte Mato-grossense, que apresentava em seu sistema de informação (Celk) um total de 370 idosos cadastrados. A referida unidade foi escolhida por contar com maior número de idosos cadastrados.

A amostra foi definida por meio de amostragem não probabilística, por conveniência. Foram inclusos na pesquisa idosos com idade igual ou superior a 60 anos, critério adotado pela Organização das Nações Unidas (ONU), que aceitaram participar voluntariamente do estudo, residentes na comunidade e cadastrados na microárea da ESF correspondente. E também aqueles que apresentaram capacidade de comunicação que permitiu a compreensão e resposta das perguntas. Foram excluídos idosos institucionalizados no período da coleta de dados. Dessa forma, o total de 50 idosos cadastrados na respectiva ESF constituiu a amostra deste estudo.

A coleta de dados foi realizada no mês de julho de 2019, por meio de entrevistas com os idosos que atenderam aos critérios de inclusão. Utilizou-se de questionários orientadores sendo o primeiro de perfil sóciodemográfico, elaborado por Sardinha e Cantanhedes (2018) e o Escore de risco de quedas elaborado por Downton (1993), traduzido e validado para o Brasil por Shiaveto (2008). O uso de instrumentos validados possibilita a produção de dados confiáveis minimizando os vieses técnicos decorrentes da má aplicação. Com as informações obtidas, houve ainda questionamentos aos participantes sobre o local de ocorrências de quedas, motivos e as principais causas do evento.

Os dados foram codificados e organizados no programa Microsoft Excel ${ }^{\circledR}$, versão 2010, de livre licença, por meio de dupla digitação para garantir a confiabilidade dos resultados. Em seguida, foram exportados para o programa software EPIINFO $^{\circledR}$, versão 6.0, para proceder à análise de dados. Aplicou-se a técnica estatística de análise descritiva, apresentada em tabelas e gráfico comparativos, com frequências absolutas e relativas, e posteriormente foi feita a discussão com base na literatura sobre o assunto pesquisado. 
Este estudo compôs um trabalho de conclusão de curso, da Universidade do Estado de Mato Grosso (UNEMAT), o qual atendeu todos os aspectos éticos e legais que envolvem os estudos com seres humanos, preconizados pela Resolução $\mathrm{n}^{\circ}$ 466/2012 do Conselho Nacional de Saúde. O projeto foi aprovado pelo Comitê de Ética em Pesquisa da Universidade Estadual de Mato Grosso em dezembro de 2018, sob o parecer CAAE: 03291118.1.0000.5166. Foram garantidos o anonimato, a privacidade e o direito à desistência em qualquer etapa da pesquisa e os envolvidos assinaram o Termo de Consentimento Livre e Esclarecido.

\section{Resultados}

Dos 50 idosos entrevistados, a maioria (74\%) era do sexo feminino e estava na faixa etária de 60 a 69 anos (44\%), $42 \%$ eram casados ou viviam em união estável, $40 \%$ moravam com familiares, 26\% deles possuíam de 1 a 4 anos de estudo e outros $26 \%$ de 9 a 10 anos de estudo. Em relação à situação ocupação, a maioria (84\%) dos idosos referiu ser aposentada ou pensionista e $46 \%$ possuíam renda mensal de até um salário mínimo.

Sobre as condições de saúde, $92 \%$ dos idosos afirmaram que não fumavam e $98 \%$ que não faziam uso de bebida alcoólica. A maioria (92\%) referiu ter problemas de saúde, sendo de maior prevalência a hipertensão (74\%), seguida da diabetes (20\%). Ao serem questionados sobre a prática de exercícios físicos, 54\% afirmaram que não realizavam nenhum tipo.

Sobre a ocorrência de quedas, constatou-se, conforme a tabela a seguir (Tabela 1), que $74 \%$ dos idosos relataram ter caído após completar 60 anos, 46\% afirmaram ter caído apenas uma vez, a maioria (73\%) mencionou que a queda sofrida foi decorrente de escorregão/tropeço. Quando questionados quanto ao local da ocorrência das quedas, 70,3\% relataram a própria residência, 37,8\% referiram fraturas como consequência das quedas. 
Tabela 1. Distribuição dos idosos quanto as variáveis relacionadas a ocorrência de quedas (N=-50). Município da Região Norte Mato-grossense, 2019.

\begin{tabular}{|c|c|c|}
\hline Variáveis & Frequência (n) & Porcentagem $(\%)$ \\
\hline \multicolumn{3}{|l|}{ Quedas após os 60 anos } \\
\hline Sim & 37 & 74,0 \\
\hline Não & 13 & 26,0 \\
\hline Total & 50 & 100,0 \\
\hline \multicolumn{3}{|c|}{ Quantidade de quedas sofridas após os 60 anos* } \\
\hline Uma queda & 17 & 46,0 \\
\hline Duas quedas & 4 & 10,8 \\
\hline Três quedas & 3 & 8,1 \\
\hline Quatro quedas ou mais & 13 & 35,1 \\
\hline Total & 37 & 100,0 \\
\hline \multicolumn{3}{|c|}{ Condição sob qual ocorreu a queda* } \\
\hline Escorregou/tropeçou & 27 & 73,0 \\
\hline Caiu da cama & 1 & 2,7 \\
\hline Tontura & 6 & 16,2 \\
\hline Caminhando & 1 & 2,7 \\
\hline Jogando bola & 1 & 2,7 \\
\hline Não soube responder & 1 & 2,7 \\
\hline Total & 37 & 100,0 \\
\hline \multicolumn{3}{|l|}{ Local de ocorrência da queda* } \\
\hline Na própria residência & 26 & 70,3 \\
\hline Ambiente externo a residência & 11 & 29,7 \\
\hline Total & 37 & 100,0 \\
\hline \multicolumn{3}{|l|}{ Consequências da queda* } \\
\hline Escoriações & 1 & 2,7 \\
\hline Hematomas & 5 & 13,5 \\
\hline Fraturas & 14 & 37,8 \\
\hline Torções & 4 & 10,8 \\
\hline Não houve danos físicos & 12 & 32,4 \\
\hline Condromalácia patelar & 1 & 2,7 \\
\hline Total & 37 & 100,0 \\
\hline
\end{tabular}

$* \mathrm{~N}=37$ (Referem-se somente aos idosos que sofreram queda). Fonte: Autores.

Referente ao risco de quedas, os resultados obtidos por meio da escala de riscos de Downton (Schiaveto, 2008), estão demonstrados na Figura 1 e mostraram que $68 \%$ dos idosos investigados possuíam alto risco para quedas. 
Figura 1. Risco de quedas dos idosos pesquisados segundo a Escada de risco de quedas de Downton. Município da Região Norte Mato-grossense, 2019.

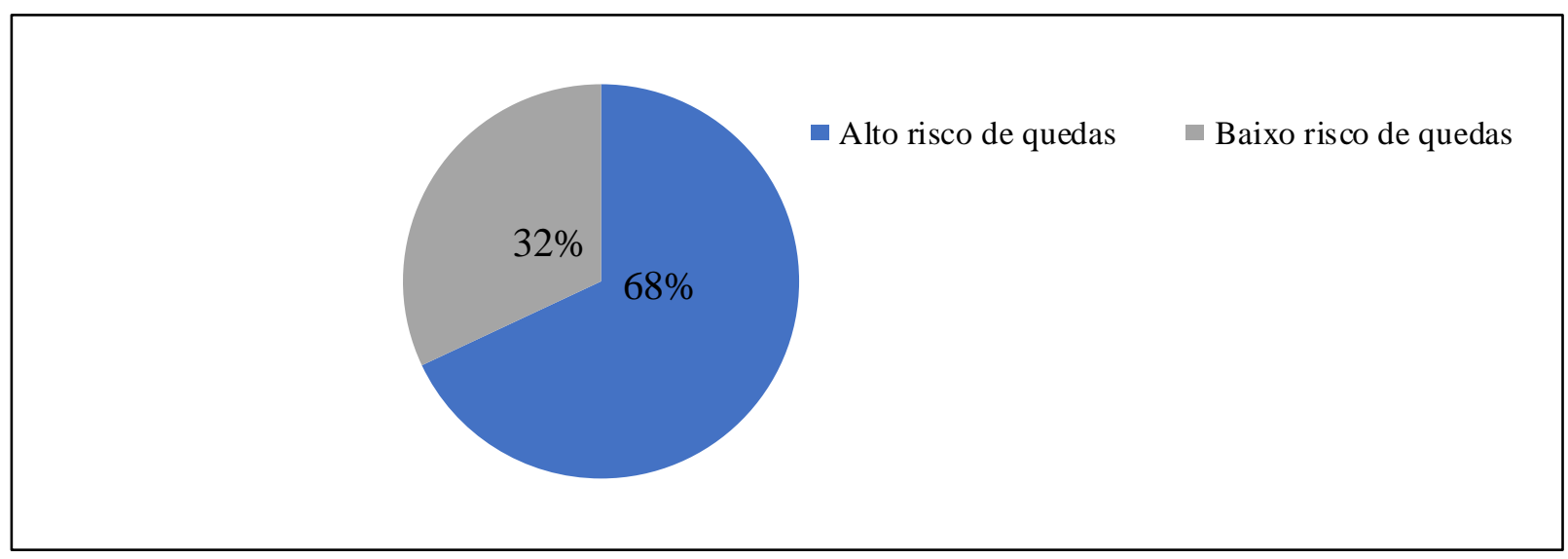

Fonte: Autores.

Por fim, os idosos foram questionados quanto as orientações sobre quedas, $72 \%$ deles relataram que já recebeu alguma orientação sobre quedas, 33,3\% afirmaram que essas orientações foram feitas por familiares, 22,2\% por conversas com amigos e somente 19,4\% afirmaram ter recebido orientações de profissionais e acadêmicos da área da saúde. Os outros idosos $(25,1 \%)$ relataram cursos/palestras, mídia e leitura como fonte de orientação para as quedas.

\section{Discussão}

Corroborando com os achados deste estudo, em que a prevalência de participantes do sexo feminino, idosos mais jovens, casada, aposentada e conta com uma renda de 1 salário mínimo foi constatada, outros estudos realizados com idosos da comunidade, encontraram características semelhantes (Nascimento \& Tavares, 2016; Cruz \& Leite, 2018).

No que se refere a escolaridade, os idosos estudados apresentaram característica muito particular, pois parte deles possuíam nível de escolaridade baixo e a mesma proporção nível mais elevado, diferente de outros estudos no qual os idosos possuíam significativamente baixo nível de escolaridade (Nascimento \& Tavares, 2016; Cruz et.al., 2018).

Em relação às condições de saúde, outros estudos encontraram características semelhantes aos dos idosos desta pesquisa, no qual, mesmo referindo problemas de saúde, possuíam bons comportamentos como estilo de vida, como não fumar e não consumir bebida alcoólica, contudo, há de se considerar que mais da metade deles, referiram sedentarismo (Abi-Ackel et.al., 2017; Ferraz; Reis \& Lima, 2017).

No que se refere as variáveis relacionadas a ocorrência de quedas, nesta pesquisa, houve um número expressivo de idosos que afirmaram ter sofrido quedas após os 60 anos, no qual, a maior parte referiu uma única queda, porém, chama atenção os idosos que relataram ter caído quatro vezes ou mais. Em outros estudos, a prevalência de quedas foi menor, variando de 33\% a 55,2\% (Guerra et.al., 2016; Chehuen Neto et.al., 2017). Ainda assim, vale ressaltar que mesmo não sendo um evento inerente ao envelhecimento, a queda está presente significativamente na vida dos idosos.

Com o avanço da idade, mesmo que o idoso tenha um envelhecimento saudável, naturalmente espera-se que aconteçam mudanças em seu organismo, ou seja, efeitos próprios do processo normal de envelhecimento. Tais mudanças implicam na fragilidade das pessoas mais velhas, podendo contribuir para o desequilíbrio, mudança na marcha, comprometimento na mobilidade, fatores estes que podem predispor a ocorrência de quedas (Macena; Hermano; Costa, 2018).

Todavia, chama a atenção, que a maioria dos idosos estudados nesta pesquisa são relativamente jovens, na faixa etária de 60 a 70 anos (Nery, 2007), fator este que contribui para a prevenção das quedas e não sua ocorrência, idosos mais velhos 
possuem maior tendência em cair. Uma possível explicação para este achado, é que em sua maioria, os idosos pesquisados são mulheres, e mesmo sendo jovens, a literatura aponta que elas geralmente possuem as maiores prevalências de quedas em relação aos idosos do sexo masculino (Nascimento \& Tavares, 2016; Souza et.al., 2017).

Outra característica que pode ter contribuído para as quedas da amostra deste estudo, é a falta de atividade física da maioria dos idosos. Autores ressaltam que a prática de exercícios físicos é um método importante para evitar o risco e ocorrência de quedas, pois ajuda no fortalecimento dos ossos, na manutenção do equilíbrio e, auxilia a força muscular. Todavia, devem ser prescritos de forma específica, considerando a singularidade de cada idoso (Mallmann et.al., 2015; Barreto; Carreira \& Marcon, 2015).

Alguns estudos mencionam que idosos sedentários tem dificuldades em manter o equilíbrio do corpo e possuem dificuldades em realizar as atividades diárias. Além disso, é importante ressaltar os benefícios da pratica de exercícios físicos, como promover maior longevidade, prevenir o declínio cognitivo, a conservação da capacidade funcional, e principalmente a redução no nível de quedas e suas consequências (Mallmann et.al., 2015; Padoin et.al., 2010; Barbosa et.al., 2017).

Quanto aos motivos que propiciaram a queda, a maioria dos idosos relataram ter escorregado/tropeçado e 16,2\% referiram tontura. Os resultados vão de encontro com os dados da pesquisa realizada por Rodrigues, Fraga \& Barros (2014) em Ribeirão Preto (SP), o qual citou como fatores intrínsecos a alteração do equilíbrio, dificuldade para caminhar, e tontura como os principais responsáveis pelas quedas.

Com relação ao local da queda, os participantes relataram ter caído principalmente na própria residência. $\mathrm{O}$ resultado do estudo corrobora com a pesquisa de Teixeira e colaboradores (2019), o qual justifica que as residências dos idosos normalmente estão irregulares, por existirem fatores de risco como pisos escorregadios e instáveis, banheiros sem corrimão, objetos espalhados pela casa e pela área, entre outros.

Além de ser preocupante a ocorrência de quedas na população estudada, outro fator importante a ser considerado, é o relato de fraturas por boa parte dos idosos como consequência das quedas. Segundo a pesquisa de Ferretti, Lunardi e Bruschi (2013), as fraturas representam umas das consequências que mais afetam a qualidade de vida dos idosos, impossibilitando-os de fazer suas atividades diárias pela redução da capacidade funcional.

Os idosos também foram avaliados quanto ao risco de quedas, cuja grande maioria apresentou alto risco para quedas, achado semelhante, porém com maior prevalência (94\%), foi encontrado em outro estudo (Reis \& Flores, 2014). O alto risco de quedas encontrado nesta população merece atenção, pois eles já são caidores e permanecem com o risco. As quedas representam deterioração global da saúde dos idosos, devido as alterações que este tipo de evento causa, como mudanças psicossociais, restrições nas atividades básicas de vida diária, medo de cair novamente que é fator de risco para a queda, perda da autoconfiança e restrições de locomoção (Reis \& Flores, 2014).

A respeito das orientações para prevenção de quedas, a maioria dos participantes relataram que receberam orientações para prevenirem as quedas. Segundo eles, sobressaiu orientações advindas dos próprios familiares, seguido de conversas com amigos e experiências. Os profissional e acadêmicos de saúde foram mencionados por pouca parcela dos idosos como fonte de orientações para as quedas.

É importante dialogar sobre o fenômeno da queda e seus fatores de risco, pois aumenta a percepção dos idosos sobre esses acidentes, sendo capaz de reduzir a exposição a seus fatores desencadeadores, direcionando esses indivíduos para que passem pelo processo de envelhecimento com qualidade (Damaceno \& Chirelli, 2019).

Com relação aos profissionais e acadêmicos de saúde, o conhecimento dos profissionais de saúde tem influenciado na execução dos métodos e/ou procedimentos que acolham as necessidades da população idosa, visto que a expectativa de vida vem crescendo. Nessa perspectiva, faz necessário rever a formação continuada das equipes multiprofissionais e até mesmo a 
formação na graduação dos profissionais, uma vez que desta forma há interferências quanto a proposição de práticas que atendam a necessidade desta população visando atender o novo perfil epidemiológico (Damaceno \& Chirelli, 2019).

Segundo o Ministério da Saúde, a ESF precisa ser um local onde acontece o acompanhamento integral a saúde desses idosos interferindo nos fatores que colocam a saúde dessa população em risco. De acordo com Oliveira e Menezes (2014) o qual realizaram um estudo com 11 enfermeiras de ESF, de um município do interior da Bahia, há a necessidade de conhecimentos específicos, por parte das enfermeiras que atuam na ESF quanto ao cuidado da pessoa idosa, ressaltando ainda a importância de capacitações frequentes. Ademais, segundo o estudo de Hilgert, Costa e Pissaia (2017), os agentes comunitários de saúde (ACS) são profissionais fundamentais para o vínculo da ESF com a população em geral, principalmente com a população idosa, uma vez que por meio das visitas domiciliares (VD) há contato direto com a realidade do ambiente que habitam, com quem vivem, dentre outros. Tais cuidados são importantes pois pessoas idosas precisam de atenção especifica e cuidados de acordo com suas reais necessidades. A ponte entre ACS, a população idosa e a ESF possibilitam um acesso completo para traçar e discutir estratégias de cuidados junto com os mesmos, visando atender a singularidade dessa população, fomentar o cuidado de forma integral e, consequentemente melhorar a qualidade de vida desta população.

Assim, é imprescindível à sensibilização da população idosa sobre os riscos que levam a queda por se tratar de um evento que gera consequências para sua saúde. Faz-se necessário incentivar a participação nas atividades para prevenção, mostrando os diversos fatores de risco e as consequências que a queda pode trazer a sua vida (Morsch; Myskiw \& Myskiw, 2016).

As limitações do estudo referem-se ao pequeno tamanho da amostra e localização da cidade atrelada a representatividade desses participantes frente às regiões metropolitanas.

\section{Conclusão}

Ao investigar o risco e a ocorrência de quedas de idosos da comunidade atendidos por uma ESF de um munícipio da região Norte Mato-grossense, foi possível observar que o risco de quedas é considerado elevado, uma vez que 68,0\% dos idosos estavam em alto risco para as quedas. Além disso, o histórico de quedas desses idosos também merece destaque, pois apresentaram um percentual de $74,0 \%$. Tais resultado são preocupantes, pois enfatizam que a queda é um evento real na vida desta população e traz a ela consequências interferindo na qualidade de vida.

O estudo possibilitou captar informações relevantes que subsidiassem o desenvolvimento da atenção à saúde do idoso considerando as peculiaridades da população no município. Os dados da pesquisa demonstraram que a temática queda precisa ser melhor trabalhada pelos profissionais enfermeiros da atenção primária com os idosos do município e faz-se necessário traçar estratégias para prevenção como educação em saúde, palestras, visitas domiciliares, dentre outros objetivando minimizar a morbimortalidade, os custos com as hospitalizações e a privação das atividades diárias da vida dos idosos da comunidade.

Além disso, é importante refletir sobre a necessidade do desenvolvimento de formações/capacitações continuadas para os integrantes da equipe da ESF, em especial o enfermeiro. Idem, a incorporação dos enfermeiros neste tipo de estudos, da área da gerontologia, enriqueceria ainda mais os achados, pois permitiria obter um panorama mais integral sobre a questão central, isto é, daqueles usuários que sofrem ou se encontram propensos a sofrer o desfecho e, complementarmente, a visão dos profissionais da saúde que realizam intervenções preventivas e/ou assistenciais.

$\mathrm{O}$ alto índice de exposição dos idosos da cidade a fatores de risco e os resultados apresentados pelo estudo objetiva implementar medidas de educação em saúde pela equipe de enfermagem da estratégia de saúde da família contribuindo para diminuição dos riscos de quedas e melhoria da qualidade de vida deste segmento populacional. Além disso, pode subsidiar e alertar quanto a necessidade de educação continuada/permanente para os profissionais enfermeiros que atuam na ESF. 
Sugere-se também, a continuidade de novas pesquisas relacionada à temática no município, devido à importância da mesma para a área da saúde, visando contribuir para o atendimento adequado e especifico da população e ao serviço dos profissionais de saúde.

\section{Referências}

Abi-Ackel, M. M., Lima-Costa, M. F., Castro-Costa, E., \& Loyola Filho, A. I. (2017). Uso de psicofármacos entre idosos residentes em comunidade: prevalência e fatores associados. Revista Brasileira de Epidemiologia, 20 (1), 57-69.

Amthauer, C., \& Falk, J. W. (2017). Discursos dos profissionais de saúde da família na ótica da assistência à saúde do idoso. Revista Online de Pesquisa: Cuidado é Fundamental, 9 (1), 99-105.

Azevedo, D. P. G. D., Azevedo, N. G., \& Istoe, R. S. C. (2018). Envejecimiento y longevidad: interfaces biológicas, psicológicas y sociales. Brasil Multicultural.

Barbosa, F. A., et.al. (2017). A influência do nível de atividade física no equilíbrio em mulheres idosas. Revista Destaques Acadêmicos, 9 (3), $197-215$.

Barreto, M. S., Carreira, L., \& Marcon, S. S. (2015). Envelhecimento populacional e doenças crônicas: Reflexões sobre os desafios para o Sistema de Saúde Pública. Revista Kairós Gerontologia, 18 (1), 325-39.

Brasil (2018). Instituto Brasileiro de Geografia e Estatística (IBGE). Projeção da População do Brasil por sexo e idade: $2010-2060$ - Revisão 2018. https://agenciadenoticias.ibge.gov.br/agencia-sala-de-imprensa/2013-agencia-de-noticias/releases/21837-projecao-da-populacao-2018-numero-de-habitantesdo-pais-deve-parar-de-crescer-em-2047

Brasil (2007). Portaria n. 3.213 de 20 de dezembro de 2007. Institui Comitê para assessorar políticas de prevenção e promoção dos cuidados da osteoporose e da queda na população idosa. http://bvsms.saude.gov.br/bvs/saudelegis/gm/2007/prt3213_20_12_2007.html

Chagas, A. M., \& Rocha, E. D. (2012). Aspectos fisiológicos do envelhecimento e contribuição da Odontologia na saúde do idoso. Revista Brasileira de Odontologia, 69 (1), 94-6.

Chehuen Neto, J. A., et.al. (2017). Fall awareness as a determining factor of this event among elderly community residentes. Geriatrics, Gerontology and Aging, 11 (1), 25-31.

Cruz, D. T., \& Leite, I. C. G. (2018). Quedas e fatores associados em idosos residentes na comunidade. Revista Brasileira de Geriatria e Gerontologia, 21 (5), $532-41$.

Cruz, D. T., Vieira, M. T., Bastos, R. R., Leite, I. C. G. (2017). Fatores associados à fragilidade em uma população de idosos da comunidade. Revista de Saúde Pública, 51 (106), 1-13.

Damaceno, M. J. C. F., \& Chirelli, M. Q. (2019). Implementação da Saúde do Idoso na Estratégia Saúde da Família: visão dos profissionais e gestores. Ciência \& Saúde Coletiva, 24 (5), 637-1646.

Ferraz, M. O. S., Reis, L. A., \& Lima, P. V. (2017). Condições de saúde de idosos portadores de Diabetes mellitus e hipertensão arterial sistêmica. Id On Line: Revista Multidisciplinar e de Psicologia, 10 (33), 794-806.

Ferreira, L. M. B. M. et. al. (2019). Quedas recorrentes e fatores de risco em idosos institucionalizados. Ciência e Saúde Coletiva, 24 (1), 67-75.

Ferretti, F., Lunardi, D., \& Bruschi, L. (2013). Causas e consequências de quedas de idosos em domicílio. Fisioterapia em Movimento, 26 (4), $753-62$.

Gasparotto, L. P. R., Falsarella, G. R., e Coimbra A. M. V. (2014). As quedas no cenário da velhice: conceitos básicos e atualidades da pesquisa em saúde. Revista Brasileira de Geriatria e Gerontologia, 17 (1), 201-209.

Guerra, H. S., et. al. (2016). Prevalência de quedas em idosos na comunidade. Revista Saúde e Pesquisa. 9 (3), 547-55.

Hilgert, M. A., Costa, A. E. K., \& Pissaia, L. F. (2017). Qualificação da assistência ao idoso na atenção básica: realização da visita domiciliar pela equipe. Revista Espaço Ciência \& Saúde, 5 (2).

Macena, W. G., Hermano, L. O., \& Costa, T. C. (2018). Alterações fisiológicas decorrentes do envelhecimento. Revista Mosaicum, 15 (27), $223-236$.

Mallmann, D. G., Galindo Neto, N. M., Sousa, S. C., \& Vasconcelos, E. M. R. (2015). Educação em saúde como principal alternativa para promover a saúde do idoso. Ciência \& Saúde Coletiva, 20 (6), 1763-1772.

Morsch, P., Myskiw, M., \& Myskiw, J. C. (2016). A problematização da queda e a identificação dos fatores de risco na narrativa de idosos. Ciência e Saúde Coletiva, 21 (11), 3565-74.

Nascimento, J. S., \& Tavares, D. M. S (2016). Prevalência e fatores associados a quedas em idosos. Texto \& Contexto Enfermagem, 25 (2), 2-9.

Nery, M (2007). Sociedade - a nova velha geração. Revista do Instituto de Pesquisa Econômica Aplicada, 32.

Oliveira, T., Baixinho, C. L., \& Henriques M. A. (2018). Risco multidimensional de queda em idosos. Revista Brasileira em Promoção da Saúde, 31 (2), 1-9.

Oliveira, M. A. S., \& Menezes, T. M. O. (2014). A enfermeira no cuidado ao idoso na estratégia saúde da família: sentidos do vivido. Revista Enfermagem UERJ, 22 (4), 513-8. 
Research, Society and Development, v. 10, n. 4, e56910414584, 2021

(CC BY 4.0) | ISSN 2525-3409 | DOI: http://dx.doi.org/10.33448/rsd-v10i4.14584

Oliveira, F. A., et. al. (2017). Estratégias educativas para promoção da saúde de idosos de um Centro de convivência. Revista Conexão UEPG, 13 (3), 500-11.

Oliveira, S. F., et. al. (2015). Produção científica acerca dos fatores de risco ambientais para quedas em idosos: revisão integrativa. Revista Enfermagem UFPE Online, 9 (2), 759-67.

Organização Mundial da Saúde (2010). Relatório global da OMS sobre Prevenção de quedas na velhice. http://bvsms.saude.gov.br/bvs/publicacoes/relatorio_prevencao_quedas_velhice.pdf

Padoin, P. G., Gonçalves, M. P., Comaru, T., \& Silva, A. M. V. (2010). Análise comparativa entre idosos praticantes de exercício físico e sedentários quanto ao risco de quedas. O Mundo da Saúde. 34 (2), 158-64.

Reis, L. A, \& Flores, C. M. R. (2014). Avaliação do risco de quedas e fatores associados em idosos. Revista Baiana de Enfermagem, 28 (1), $42-9$.

Rodrigues, I. G., Fraga, G. P., \& Barros, M. B. A. (2014). Quedas em idosos: fatores associados em estudo de base populacional. Revista Brasileira de Epidemiologia, 17 (3), 705-18.

Sardinha, A. H. L., \& Cantanhêdes, N. L. C. (2018). Quedas em idosos: avaliação dos fatores de riscos. Revista Nursing, 21 (240), $2160-63$.

Schiaveto, F. V. (2008). Avaliação do risco de quedas em idosos da comunidade. Dissertação (Mestrado em Enfermagem) - Universidade de São Paulo. Ribeirão Preto. http://10.11606/D.22.2008.tde-19122008-153736.

Souza, L. H. R., Brandão, J. C. S., Fernandes, A. K. C., \& Cardoso, B. L. C. (2017). Queda em idosos e fatores de risco associados. Revista de Atenção à Saúde, 15 (54), 55-60.

Teixeira, D. K. S., Andrade, L M., Santos, J. L. P., \& Caires, E. S. (2019). Quedas em pessoas idosas: restrições do ambiente doméstico e perdas funcionais. Revista Brasileira de Geriatria e Gerontologia, 22 (3), 1-10.

Veras, R. P., e Oliveira, M. (2018). Envelhecer no Brasil: a construção de um modelo de cuidado. Ciência e Saúde Coletiva, 23(6), 1929-36.

World Health Organization (2012). Falls. Fact sheet $N^{\circ} 344$. http://www.who.int/mediacentre/factsheets/fs344 /en/

Zangirolami-Raimundo, J., Echeimberg, J. O., \& Leone, C. (2018). Research methodology topics: cross-sectional studies. Journal of Human Growth and Development, 28 (3), 356-60. 